\title{
A knowledge-based system for end mill selection
}

\author{
Prasad, K. ${ }^{a}$, Chakraborty, S. ${ }^{\mathrm{a},{ }^{*}}$ \\ ${ }^{a}$ Department of Production Engineering, Jadavpur University, Kolkata, India
}

\section{A B S T R A C T}

In the present global competitive environment, manufacturing organizations are being forced to constantly develop newer methods/technologies for producing high quality products/components at the minimum possible cost to satisfy the diverse and dynamic needs of customers. Selection of a proper cutting tool within a process planning system is vital for the productive efficiency and cost effectiveness of a manufacturing process. In this paper, a knowledgebased system is developed in Visual BASIC 6.0 and subsequently implemented for selection of an appropriate end mill for a given machining application from a set of feasible alternatives. Although, there are some published research papers on the applications of knowledge-based systems for selecting of cutting tools, none of them has investigated its scope for choosing a suitable end mill from a comprehensive list of options available on the market. The developed system first narrows down the list of end mills based on some predefined parameters as set by the process planner and then ranks the feasible end mills according to their suitability for the desired machining application. While ranking the end mill alternatives, criteria weights are determined using Shannon's entropy method to avoid subjectivity in judgments. It also guides the process planner in identifying the corresponding speed and feed for different combinations of workpiece material and machining operation.
\end{abstract}

\section{ARTICLE INFO}

Keywords:

End mill

Decision making

Knowledge-based system

Entropy method

Rank

*Corresponding author:

s_chakraborty00@yahoo.co.in

(Chakraborty, S.)

Article history:

Received 1 September 2015

Revised 21 January 2016

Accepted 25 January 2016 


\section{References}

[1] Rubio, L., De la Sen, M. (2006). Selection of mill cutter and cutting parameters through an expert system, Advances in Computer, Information, System Sciences, Engineering, Springer, Netherlands, doi: 10.1007/1-4020-5261-8 2.

[2] Rao, P.N., (2009). Manufacturing Technology, (3rd edition), Tata McGraw-Hill Publishing Company Limited, New Delhi, India.

[3] Klancnik, S., Ficko, M., Balic, J., Pahole, I. (2015). Computer vision-based approach to end mill tool monitoring, International Journal of Simulation Modelling, Vol. 14, No. 4, 571-583, doi: 10.2507/IJSIMM14(4)1.301.

[4] Reddy, N.S.K., Rao, P.V., (2005). Selection of optimum tool geometry and cutting conditions using a surface roughness prediction model for end milling, International Journal of Advanced Manufacturing Technology, Vol. 26, No. 11, 1202-1210, doi: 10.1007/s00170-004-2110-y.

[5] Li, L.L., Zhang, Y.F. (2006). Cutter selection for 5-axis milling of sculptured surfaces based on accessibility analysis, International Journal of Production Research, Vol. 44, No. 16, 3303-3323, doi: 10.1080/00207540500444720.

[6] Novak-Marcincin, J., Janak, M., Novakova-Marcincinova, L., Fecova, V. (2012). Possibility of a quick check on milling strategy suitability, Tehnički vjestnik - Technical Gazette, Vol. 19, No. 4, 959-964.

[7] Arezoo, B., Ridgway, K., Al-Ahmari, A.M.A. (2000). Selection of cutting tools and conditions of machining operations using an expert system, Computers in Industry, Vol. 42, No. 1, 43-58, doi: 10.1016/S0166-3615(99)00051-2.

[8] Carpenter, I.D., Maropoulos, P.G., (2000). Automatic tool selection for milling operations Part 1: Cutting data generation, Proceedings of the Institution of Mechanical Engineers, Part B: Journal of Engineering Manufacture, Vol. 214, No. 4, 271-282, doi: 10.1243/0954405001517667.

[9] Sorby, K., Tonnessen K. (2000). Methodology for selection of cutting tool and machining data for high speed flank milling, In: Proceedings of the $2^{\text {nd }}$ International Seminar on Improving Machine Tool Performance, La Baule, France, from http://imtp.free.fr/imtp2/C1/Sorby Knut revised.pdf, accessed September 1, 2015.

[10] Čuš, F., Balič, J. (2001). Selection of cutting conditions and tool flow in flexible manufacturing system, Journal of Materials Processing Technology, Vol. 118, No. 1-3, 485-489, doi: 10.1016/S0924-0136(01)00988-8.

[11] Edalew, K.O., Abdalla, H.S., Nash, R.J. (2001). A computer-based intelligent system for automatic tool selection, Materials \& Design, Vol. 22, No. 5, 337-351, doi: 10.1016/S0261-3069(00)00106-0.

[12] Wang, X., Da, Z.J., Balaji, A.K., Jawahir, I.S. (2002). Performance-based optimal selection of cutting conditions and cutting tools in multipass turning operations using genetic algorithms, International Journal of Production Research, Vol. 40, No. 9, 2053-2065, doi: 10.1080/00207540210128279.

[13] Zhao, Y., Ridgway, K., Al-Ahmari, A.M.A. (2002). Integration of CAD and a cutting tool selection system, Computers \& Industrial Engineering, Vol. 42, No. 1, 17-34, doi: 10.1016/S0360-8352(01)00061-4.

[14] Byrne, G., Dornfeld, D., Denkena, B. (2003). Advancing cutting technology, CIRP Annals - Manufacturing Technology, Vol. 52, No. 2, 483-507, doi: 10.1016/S0007-8506(07)60200-5.

[15] Muršec, B., Čuš, F. (2003). Integral model of selection of optimal cutting conditions from different databases of tool makers, Journal of Materials Processing Technology, Vol. 133, No. 1-2, 158-165, doi: 10.1016/S0924-0136 (02)00226-1.

[16] Oral, A., Cakir, M.C. (2004). Automated cutting tool selection and cutting tool sequence optimisation for rotational parts, Robotics and Computer-Integrated Manufacturing, Vol. 20, No. 2, 127-141, doi: 10.1016/i.rcim. 2003.10.006.

[17] Svinjarević, G., Stoić, A., Kopač, J. (2007). Implementation of cutting tool management system, Journal of Achievements in Materials and Manufacturing Engineering, Vol. 23, No. 1, 99-102.

[18] Wang, X., Da, Z.J., Balaji, A.K., Jawahir, I.S. (2007). Performance-based predictive models and optimization methods for turning operations and applications: Part 3 - Optimum cutting conditions and selection of cutting tools, Journal of Manufacturing Processes, Vol. 9, No. 1, 61-74, doi: 10.1016/S1526-6125(07)70108-1.

[19] Arshad, H., Hassan, R., Omar, N., Sahran, S. (2010). Virtual cutting tool management system for milling process, International Journal of Computer Science and Network Security, Vol. 10, No. 2, 148-153.

[20] Ostojic, G., Tadic, B., Luzanin, O., Stankovski, S., Vukelic, D., Budak, I., Miladinovic, L. (2011). An integral system for automated cutting tool selection, Scientific Research and Essays, Vol. 6, No. 15, 3240-3251.

[21] Vukelic, D., Tadic, B., Jocanovic, M., Luzanin, O., Simeunovic, N., (2011). A system for computer-aided selection of cutting tools, ACTA Technica Corviniensis - Bulletin of Engineering, Vol. 4, No. 3, 89-92.

[22] Chougule, P.D., Kumar, S., Raval, H.K., (2014). An expert system for selection of carbide cutting tools for turning operations, In: Proceedings of $5^{\text {th }}$ International \& $26^{\text {th }}$ All India Manufacturing Technology, Design and Research Conference, India, (252-1)-(252-6).

[23] Rao, R.V. (2013). Decision making in the manufacturing environment using graph theory and fuzzy multiple attribute decision making methods, Volume 2, Springer Series in Advanced Manufacturing, Springer-Verlag, London, UK, doi: 10.1007/978-1-4471-4375-8. 


\section{APEM}

Advances in Production Engineering \& Management

Letnik 11 | Številka 1 | Marec 2016 | Strani 15-28

http://dx.doi.org/10.14743/apem2016.1.206
ISSN 1854-6250

Spletna stran: apem-journal.org Izvirni znanstveni članek

\title{
Na znanju temelječ system za izbiro rezkala
}

\author{
Prasad, K. ${ }^{a}$, Chakraborty, S. ${ }^{\text {a, }}$ \\ ${ }^{a}$ Department of Production Engineering, Jadavpur University, Kolkata, India
}

\section{POVZETEK}

Podjetja morajo zaradi globalne konkurenčnosti poslovnih okolij neprestano razvijati nove metode in tehnologije (ob čim manjših stroških) pri izdelovanju visokokakovostnih izdelkov, da zadovoljijo potrebe kupcev in zahteve po raznolikosti ponudbe. Izbira ustreznega orodja pri načrtovanju procesov igra vitalno vlogo, da se doseže učinkovitost proizvodnje in stroškovna vzdržnost. $\mathrm{V}$ delu smo s pomočjo orodja Visual BASIC 6.0 razvili in uporabili na znanju temelječ sistem za izbiro ustreznega rezkala izmed veliko alternativnih možnosti. Čeprav nekatere raziskave $\mathrm{v}$ dostopnih virih navajajo aplikacije na znanju temelječih sistemih za izbiro rezalnih orodij, pa nobena ne omogoča izbiro ustreznega orodja iz obširnih seznamov orodij, ki so na trgu. V članku razvit sistem najprej na osnovi v naprej določenih parametrov zoži seznam mogočih rezkalnih orodij, nato pa jih rangira z ozirom na sposobnost izvedbe želenega obdelovalnega postopka. Da bi se izognili subjektivni presoji o ustreznosti orodja, je med rangiranjem alternativ (tj. možnih izbir orodja) uporabljena Shannonova entropijska metoda za utežitev kriterijev, ki prav tako pomaga načrtovalcu procesov izbrati ustrezne obdelovalne hitrosti in podajanja za različne kombinacije obdelovancev in obdelovalnih operacij.

\section{PODATKI O ČLANKU}

Ključne besede:

Rezkalo

Odločitvene strategije

Na znanju temelječ sistem

Entropijska metoda

Rang

* Kontaktna oseba:

s_chakraborty00@yahoo.co.in

(Chakraborty, S.)

Zgodovina članka:

Prejet 1. septembra 2015

Popravljen 21. januarja 2016

Sprejet 25. januarja 2016 